\title{
Violencia escolar o violencia educativa: el dilema no planteado desde el profesorado
}

\section{School violence or educational violence: a dilemma not confronted by teachers}

DOI: https://doi.org/10.32870/dse.v0i24.1046

\author{
Rodolfo Cruz Vadillo* \\ Emma Verónica Santana Valencia** \\ Paulina Iturbide Fernández ${ }^{* * *}$
}

\begin{abstract}
Resumen
Este texto es fruto de una serie de trabajos realizados en el marco del proyecto "Inclusión educativa, familiar y social", de un grupo de investigación cuyo propósito ha sido analizar los procesos de inclusión y exclusión desde las instituciones escolares. Tiene como objetivo analizar los significados de la violencia escolar a partir de las construcciones que han realizado profesores de enseñanza media en México. Fue un estudio cualitativo de corte descriptivo cuya técnica fue el cuestionario cualitativo, que fue aplicado a 57 profesores de bachillerato del estado de Puebla. Dicho instrumento tuvo como propósito recopilar información sobre los significados que los profesores tenían acerca de la violencia escolar y las posibilidades de la acción pedagógica para reducirla. Las conclusiones arrojan la necesidad de profundizar acerca de la violencia educativa, la cual es aquella que está presente en los espacios escolares y se personaliza en daños o perjuicios hacia la comunidad, pero también, en la propia violencia que se ejerce desde las estructuras escolares al no ser capaces de cuestionar lo establecido y reconocer lo dilemático del fenómeno.
\end{abstract}

Palabras clave: violencia escolar - estudiantes - educación - juventud - inclusión.

\section{Abstract}

This paper is the result of a series of work conducted within the framework of the project "Educational, family and social inclusion" of a research team whose aim is to analyze the processes of inclusion and exclusion from school. The paper analyzes the meanings of school violence based on the constructions made by high school teachers in Mexico through a qualitative, descriptive study. We used a qualitative

* Doctor en Investigación Educativa. Líneas de investigación: políticas educativas sobre inclusión educativa y discapacidad. SNI 1. ProfesorInvestigador. Universidad Popular Autónoma del Estado de Puebla (UPAEP). México. rodolfo.cruz@upaep.mx

** Doctora en Ciencias para la Familia. Línea de investigación: familia, resiliencia e inclusión. Miembro de la Red Internacional de Investigadores y Participantes sobre Integración Educativa (RIIE) y del Cuerpo Académico de Profesionalización Docente. Profesora-investigadora y directora académica del Posgrado en Orientación y Desarrollo Familiar. Universidad Popular Autónoma del Estado de Puebla (UPAEP). México.emmaveronica.santana@upaep.mx

*** Doctora en Educación. Líneas de investigación: educación inclusiva, ética profesional docente y formación del profesorado. Miembro de la Red Nacional de Educación y Valores (REDUVAL). Directora Académica de las Licenciaturas en Psicopedagogía y Pedagogía e Innovación Educativa. Universidad Popular Autónoma del Estado de Puebla (UPAEP). México. paulina.iturbide@upaep.mx 
questionnaire that was answered by 57 high school teachers from the state of Puebla, Mexico, with 7 open questions to collect information about the meanings that teachers had about school violence and the possibilities of pedagogical action to reduce it. Among our conclusions is the need to think about educational violence, which is present in school spaces and materializes as damages towards the community, as well as the violence exerted through school structures due to the inability to question what is established and recognize the dilemma of the phenomenon.

Keywords: school violence - students - education - youth - inclusion.

\section{Introducción}

Actualmente existen en el mundo 1,200 millones de jóvenes entre 15 y 24 años de edad (Encuesta de Jóvenes en México, 2019), lo que ciertamente hace pensar que el tema de la juventud no es una situación que pueda considerarse minúscula, menos aún cuando simbólicamente articula una gran cantidad de visiones, dimensiones y explicaciones en su haber. Según datos de la CEPAL (Trucco y Ullmann, 2015), se estima que aproximadamente 30 millones de jóvenes en América Latina se encuentran desvinculados del sistema educativo. En México, de acuerdo con el INEGI (2015), viven 30.7 millones de jóvenes de entre 15 y 29 años, los cuales representan $24.6 \%$ de la población. Del total de la población de jóvenes en el país, la mitad están estudiando (50.2\%). No obstante, los jóvenes que se encuentran dentro de los sistemas educativos no están ajenos a las consecuencias de las desigualdades y las violencias estructurales que los han colocado en un terreno vulnerable. Lo anterior permite visibilizar la complejidad del problema de dicha violencia, las articulaciones entre exterioridad e interioridad y las formas sutiles, simbólicas y materiales, desde las cuales la convivencia en el mundo y las instituciones que se han constituido para promoverla, representan hoy uno de los mayores retos. Es en este espacio donde también es necesario interrogar las relaciones que se construyen con los jóvenes desde lo escolar, sobre todo con aquellos agentes encargados de llevar a cabo el proyecto ético-político de la nación. Son los profesores, sus significados y creencias lo que interesa en este trabajo, puesto que el posicionamiento del mismo nos lleva a cuestionar ese exterior constitutivo desde el cual se afirma o niega lo joven y desde el cual se construyen las posibilidades para ser, estar y habitar este mundo, en específico el espacio escolar.

Este texto es fruto de una serie de trabajos realizados en el marco del proyecto "Inclusión educativa, familiar y social", promovido por un grupo de investigación cuyo propósito ha sido analizar los procesos de inclusión y exclusión desde las instituciones escolares. En dichos espacios de reflexión se ha hecho presente de forma colateral el tema de la violencia escolar, puesto que para poder hablar de procesos inclusivos se requiere un tipo de participación por parte de los diferentes agentes de la comunidad, entre ellos, los estudiantes; participación capaz de 
formularse en clave de convivencia, permitiendo la constitución de verdaderos espacios inclusivos. Sin embargo, cuando se ha intentado llevar a cabo acciones específicas para llegar a construir comunidades inclusivas con estudiantes, sobre todo con jóvenes, los retos y dificultades no se han hecho esperar. Señalando que la complejidad del acto implica, de entrada, una mirada comprensiva del fenómeno, así como la visibilización de las relaciones con otros elementos presentes, es así como la tríada inclusión-juventud-violencia, parece conjugar dichos retos.

En este marco, las políticas públicas sobre la juventud parecen ubicarse a lo largo y ancho de las latitudes planetarias, atravesando los espacios institucionalizados, haciendo visible el problema ahí donde el intento de estar juntos cada día representa el horizonte deseable de llegada. Las posiciones, imbricaciones, trasposiciones y demás formulaciones de política posibles, representan los colores y tonalidades que pueden habitar en las propuestas y ordenamientos de estado y gobierno. Lo cierto es que, sea cual sea el énfasis u orientación, la juventud preocupa. Su situación de vulnerabilidad, que no señala una condición sino más bien una relación, lleva a pensar en el posible peligro que corren los jóvenes si la omisión, la invisibilización y la indiferencia logran habitar sus contextos de interacción, en este caso, si la reflexión y la mirada crítica no se hace presente en la institución escolar, lugar donde posiblemente algo hay por hacer frente a esta situación. Es en este punto donde la figura del profesor cobra sentido y posibilita reflexionar sobre la problemática; es el acto educativo una relación que se ejercita día a día y desde la cual se constituyen puentes para la convivencia o se emplaza a la violencia, una relación desde lo pedagógico sin dejar de ver lo ético.

Como se ha señalado, el propósito de este estudio es analizar los significados de la violencia escolar que han construido los profesores de enseñanza media en una zona escolar del estado de Puebla, México. Este trabajo se justifica desde varios niveles, uno de ellos es político, pues pretende ayudar a visibilizar las complejas relaciones que se tejen en los espacios escolares en torno al fenómeno de la violencia y, con ello, coadyuvar a repensar el papel político que tiene la escuela, sus lógicas y racionalidades. Por otra parte, se justifica en lo social, pues el tema de la violencia no es una preocupación que se da en la escuela y desde la escuela; fuera de los márgenes de las instituciones coexisten otras formas de violencia que se traslapan y articulan, y al hacerlo, dificulta ubicar sus márgenes. Asimismo, este trabajo tiene una justificación educativa, pues sirve para realizar una autocrítica a las intervenciones que se realizan desde el margen escolar, que si bien abogan con un concepto dialógico de convivencia, escasamente han cuestionado el papel de la propia institución y la flexibilidad para la participación de los estudiantes como sujetos políticos con voluntades e intereses, que muchas veces desbordan lo que los curricula escolares plantean y lo que la mirada del adulto posibilita para con la juventud. Desde una mirada ética, se plantea un análisis sobre las tensiones individuales y estructurales de la violencia, que posibilita una aproximación también moral y valoral, además de lo pedagógico, sobre lo que las acciones y reacciones de los agentes que ejercen la violencia suponen. 


\section{La acción educativa y la violencia escolar: efectos y producciones}

En el ámbito educativo existe una diversidad de trabajos que han abordado el tema de lo escolar y, con ello, las conductas y modos de estar que se consideran deseables en toda acción y relación pedagógica. Una de las vetas que se pueden observar, responde a una serie de articulaciones y recurrencias que se presentan entre el tema de la violencia escolar y la inclusión educativa en jóvenes estudiantes; específicamente, cuando se trastocan los problemas educativos relacionados con sus actitudes y sus conductas. En otras palabras, las articulaciones entre violencia-inclusión-juventud se dan en el mismo momento que se busca la asimilación de las normas y formas de convivencia escolar por parte de los estudiantes, y se hace presente en la acción pedagógica por parte de profesores y directivos.

En este marco, se pueden señalar numerosos estudios que han emplazado la necesidad de medidas específicas y propuestas de intervención sobre las conductas de los estudiantes, cuyo centro de interés y blanco de análisis responde a una mirada esencialista del problema (Justicia-Arráez, Alba, Fernández, Justicia, 2012; García, Armas, 2008; Salgado, Fuentes, Escobar, 2009; Romero, Benavides, Quesada, Álvarez, 2016; Fajardo, Fajardo, 2010; Abella, Barrio, Manga, 2006; Pastor, 2016). Las investigaciones, en este tenor, han señalado una gran diversidad de abordajes en donde las respuestas y críticas a estas problemáticas intentan trabajar desde el plano individual, asumiendo que sus causas son de índole privada, es decir, particular, por tanto, basta con aplicar una serie de estrategias a los estudiantes para que se inicie la transformación de su "identidad".

Por otra parte, existen trabajos cuyo foco de interés está puesto en las dinámicas familiares y elementos contextuales cercanos al estudiante (Mayorga, Godoy, Riquelme, Ketterer, Gálvez, 2016; Gracia, Fuentes, García, 2010), donde es posible explicar las conductas antisociales que muchos jóvenes expresan, por ejemplo, en los espacios escolares. Sin embargo, dichos trabajos se limitan nuevamente a ubicar el problema en lugares, sujetos y estructuras que son ajenos a la escuela y sus lógicas, y aunque posibilitan el diálogo en las dinámicas estudiadas, al final sólo dan cuenta de una situación en donde los mecanismos de escolarización escasamente son visibles como elementos nodales del mismo y, por ende, la responsabilidad parece seguir estando del lado de los estudiantes, sus familias y la comunidad en donde viven.

Cabe señalar que todos estos trabajos permiten comprender el problema desde los procesos individuales y más privados de los infantes o adolescentes, lo cual, sin duda representa aportes significativos para su análisis y el diseño de algunas propuestas para su solución. Sin embargo, también es cierto que muchas veces, cuando la mirada enfoca demasiado una particularidad, puede perder de vista otros elementos que circulan y que participan intersticialmente en dicha problemática, facilitando una serie de explicaciones que terminan colocando al infante $o$ joven en el terreno del déficit, de la enfermedad y la patología y, con ello, el problema queda lejos del espacio educativo, de la convivencia y la relación social y más cercano a un tema de salud. 
Vasen (2010) ha señalado la psiquiatrización y patologización en la que se ha visto sumida la infancia y la adolescencia al ser, en casos extremos, la medicina el recurso central para cambiar las conductas "violentas" de los estudiantes. Si bien no se niega la existencia de determinadas problemáticas en donde la medicación puede ser una opción para la mejora del bienestar del estudiante, la crítica más bien es hacia la instauración de estas lógicas de razonamiento que pretenden explicar toda conducta "desviada" como parte de un cuadro sintomático que representa estados individuales que requieren una intervención igualmente individual. En otras palabras, estas racionalidades no sólo se han quedado en el terreno de la educación especial (con sus cuadros diagnósticos) y de la mirada biomédica que la sostiene, también han transitado a otros espacios simbólicos que ya no permiten una fácil delimitación entre la presencia de síndromes y problemáticas que aquí, siguiendo a Solé y Moyano (2017), refiere a una especie de colonización "Psi" del discurso educativo, donde el uso abusivo de una serie de diagnósticos psicológicos relacionados con la salud mental, han posibilitado una patologización de los estudiantes.

En línea con lo anterior, Pérez-Sevilla (2017) señala cómo la presencia de la lógica hegemónica de la salud pública ha significado la violencia como un proceso que se materializa en la individuación de la misma. La visión de la enfermedad, que muchas veces está presente en las racionalidades que guían las propuestas de intervención, si bien no pueden considerarse erróneas, tienden a invisibilizar las cuestiones estructurales y culturales de la violencia (Galtung, 1990). En este sentido, Skliar (2005) ha abordado reiteradamente estos procesos de psicologización y patologización de las infancias y las juventudes, dando cuenta de los mecanismos que han construido tal ejercicio de gubernamentalización de las escuelas, en donde existen dispositivos que premian el tratamiento individual y niegan las relaciones complejas que muchas veces subyacen a los actos sobre violencia que hemos señalado.

Por su parte, Dubet (1998) ha insistido que en muchos casos la violencia puede ser mera reactividad de los estudiantes frente a un proceso educativo que escasamente tiene sentido para ellos mismos, ejerciendo lo que Bourdieu y Passeron (1995) llaman violencia simbólica, es decir, una imposición de ciertas estructuras cognitivas objetivas que se muestran como verdaderas y legítimas y cuya acción pedagógica representa la mayor estrategia.

En línea con lo anterior, Fierro y Carbajal (2019) han dado cuenta en diversos estudios del papel que juega la normativa escolar en exacerbar la violencia al interior de las instituciones; en otras palabras, estas investigadoras comentan que muchas veces la utilización del reglamento escolar como piedra angular de la disciplina ha permitido que, lejos de erradicar el problema, éste se haga presente con mayor intensidad pues se reconoce que, si bien dicho instrumento puede estar claramente concretado en forma de normas específicas, su interpretación y traducción permite una cierta plasticidad que en muchos de los casos no posibilita un uso justo del mismo. 
El hecho es que la violencia en la escuela representa una fuerte problemática que ocupa y preocupa a un gran número de educadores; sin embargo, no se ha encontrado una estrategia que permita de una vez y para siempre erradicarla. Lo anterior tiene que ver con su relación con la violencia estructural, política y social (Treviño, 2020) que se hace presente en los contextos, y de la cual la escuela no es ajena. Gran parte de los hechos violentos que la escuela presencia pueden considerarse reflejo de problemáticas macro que se materializan en las relaciones e interacciones cotidianas, bajo la tesis de una existencia de condiciones de violencia en lo social, sesgadas por la precariedad, la pobreza y la desigualdad, mismas lógicas visibles desde los espacios educativos $y$, sobre todo, reflejo de ellas.

\section{Ser joven: algunos significados}

Según la Organización de las Naciones Unidas, hablar de juventud remite, entre muchos otros aspectos, a un periodo del desarrollo humano que comprende entre los 15 y 24 años de edad. Para muchos es un periodo que se encuentra en el tránsito entre la niñez y la adultez y en donde la búsqueda de la identidad y sentido de vida parecen ser una parte medular. Sin embargo, formular una definición que coloque aspectos ontológicos y ónticos de lo que implica el "ser joven", puede resultar una tarea compleja para cualquier estudioso del tema; ser joven puede ser explicado con una amplia diversidad de miradas disciplinares, teóricas e históricas (Villa, 2011) que dan cuenta de una dispersión referencial.

Por un lado, el concepto de juventud hace referencia a un estadio de la vida humana caracterizado muchas veces por la falta de experiencia, de maduración, incapacidad de autonomía, facilidad de manipulación, excesos, indiferencia, entre otros calificativos; adjetivaciones que han colocado al joven como un sujeto de riesgo, incluso peligroso (Mansilla, 2000). Esta mirada, en algunos casos, ha traído consigo la estigmatización de esta etapa de vida y la colocación de las preocupaciones sobre los jóvenes cuya supuesta "falta" de experiencia y capacidad los ha llevado a habitar espacios en donde su participación se ha reducido al seguimiento de reglas y normas y a la sujeción de discursos que intentan "corregir" su camino, llevándolo a ser y estar bajo la dictadura de la mirada del adulto, el cual es el que debe decidir sobre el presente y futuro del joven. Espacios donde el diálogo, las propuestas, la agencia y el reconocimiento parecen ubicar posiciones precarias e incluso inexistentes.

Por otro lado, la referencia a la juventud responde a una serie de representaciones que la piensan en potencia, es decir, no sólo lo es, de acuerdo a sus méritos y esfuerzos, sino a lo que puede llegar a ser si el contexto le ofrece la cantidad de apoyos necesarios (Villa, 2011). En este sentido, esta visión representa una mirada que se enfoca en la posibilidad y capacidad a partir de las acciones, los puentes y los andamios que requieran ser construidos alrededor del joven mismo. En otras palabras, la invitación a repensar lo joven, no como espacio de la inestabilidad y la falta, no como materialización de inmadurez e inconsciencia, sino como la aspiración, el 
deseo y la voluntad de pensarlo como condición de posibilidad para la transformación y cambio social. No obstante, la mirada hacia el joven sigue siendo algo en falta, lo que todavía no es, y por ende, la necesidad de intervenciones que permitan conducirlo a estadios de mayor estabilidad y desarrollo.

\section{Juventud, violencia e intervenciones educativas}

En la actualidad, la manifestación de la violencia en la Educación Media Superior (EMS) es un fenómeno que en los últimos años ha sido una constante, la cual se presenta bajo diversas condiciones y es protagonista de la realidad cotidiana. El interés en el conocimiento y estudio de la violencia escolar, en varios países se muestra como un síntoma de los tiempos actuales, convirtiéndose en un problema para abordar desde la salud, los derechos humanos y ámbitos formativos, particularmente la escuela. Estudiosos del tema han observado que este fenómeno es un reflejo de lo que sucede en la sociedad, además de que es promovida por los medios de comunicación, las redes sociales y la cultura, encontrando en la escuela un espacio fecundo donde se reproduce a través del ejercicio del poder, el dominio de las autoridades, discriminación, agresiones físicas, amenazas, lenguaje verbal ofensivo entre los alumnos, así como el contexto social conflictivo en el que esté inmersa la institución educativa (Saucedo, Guzmán, 2018; Aldana, Seáñez, 2017; Del Tronco, Madrigal, 2013). Varios son los hechos que dejan evidencia de los fenómenos violentos desde una mirada sistémica, donde la función escolar responde a mecanismos de dominación instaurados por la misma institución, a partir de la cual se manifiesta una forma de violencia simbólica y emocional (Chuquilin, Zagaceta, 2017) que puede llegar a criminalizar a los estudiantes (Saucedo, Guzmán, 2018), haciendo de esto un fenómeno para profundizar desde diversas miradas.

En este posicionamiento, Gómez, Zurita y López (2013) consideran que la violencia en las escuelas se puede comprender desde tres elementos esenciales:

- Como un recurso de poder establecido por el profesor y las autoridades escolares para ejercer un control y dominar.

- Entre los pares, donde se ejerce de manera horizontal, como una fuerza evidente o escondida, con el fin de obtener algo.

- $Y$, finalmente, como una práctica de la propia escuela que busca preservar, a través de unas normas formales o informales, el orden, el control y la disciplina.

La violencia es una construcción social que repercute formando al sujeto que designa. Esto indica que se construye a través de la observación directa de las expresiones de violencia y de las acciones concretas en donde se manifiestan. Además, existen factores interrelacionados con la integración de los estudiantes a su comunidad educativa, a las diversas condiciones de 
socialización generadas desde el sistema familiar y la propia actitud del alumno, donde se impone de manera arbitraria una fuerza del exterior sobre la víctima que la experimenta (Míguez, $2007,2009,2012)$. En varias ocasiones, la función escolar responde a formas de dominación instauradas para mantener un gobierno, al cual los estudiantes deben adaptarse. En ocasiones, se observan acciones tiránicas de los docentes o directores donde la discriminación y hechos denigrantes configuran el proceso de enseñanza-aprendizaje y conforman un espacio de riesgo desde la escuela (Del Tronco, Madrigal, 2013).

En este marco, el tema de la violencia escolar parece hacerse más virulento en la medida que se traslapa con la entrada de la adolescencia y la juventud, y se articula con espacios institucionalizados como la escuela. Desde la mirada del desarrollo humano, la adolescencia y la juventud se consideran etapas críticas donde el joven está en la búsqueda de su identidad y, por ende, tiende a cuestionar lo establecido (Colom, Fernández, 2009). En este sentido, la acción pedagógica puede optar por diversos caminos. Uno de ellos podría ser la intervención educativa, de tal suerte que los jóvenes puedan ajustarse a lo que está instituido, es decir, a las lógicas y verdades que desde el mundo del adulto se muestran a través de dicho proceso. Por otro lado, la acción podría partir de las realidades y sentidos del propio estudiante con la finalidad de que, más allá de llevarlo a procesos de autorregulación que terminen en el disciplinamiento de los cuerpos (Foucault, 2002), permitan construir puentes como apoyos (Aznar, 2008) para que sean, en la medida de lo posible, los propios estudiantes quienes inicien el complejo camino hacia la toma de decisiones que los llevarán a generar espacios de libertad que, al final, puedan traducirse en compromisos más potentes y profundos para con la comunidad en donde viven.

El problema en este punto es que muchas veces las respuestas educativas transitan entre ambos polos de la situación. En dichas formas de intervención y abordaje se pierde de vista que, aunque se dialogue con el estudiante y se intente responder a sus intereses y necesidades, hay una visión que se presenta como el único horizonte de llegada, es decir, las propuestas curriculares han sugerido estas formas de acción situada y creativas del profesor desde lo curricular, sin embargo, sea cual sea el camino a seguir, el lugar de llegada está marcado por el mismo plan de estudios y los valores sociales y culturales hegemónicos de la época. Por ejemplo, lo que señala el perfil de egreso del propio estudiante, delimita el margen de acción y coloca visiones de lo que significa ser adulto en determinado espacio. Al final, al estudiante que no logra llegar a este puerto, posiblemente le espera el destierro, para muchos, la "autoexclusión" de los sistemas escolares, puesto que presentan escasa disponibilidad de regulación y aceptación a las normas de la adultez, normas que definitivamente ya estaban marcadas de inicio, haciéndolo pensar que, si bien se habían dispuesto espacios para el diálogo, todo podría ser un montaje que al final lo llevará por el camino correcto sin importar lo que se pensara o lo convencido que se estuviera de lo contrario. 


\section{El dilema: violencia escolar, violencia educativa}

Como se puede observar, el escenario planteado representa un entramado complejo en torno al tema de la violencia; por un lado, hay una serie de ordenamientos y procesos de gubernamentalización escolar que deben llevar inevitablemente al disciplinamiento de los cuerpos, pero, por otro lado, hay una serie de resistencias a dicha acción que se materializa en categorías como la violencia escolar, y que posibilitan pensar y señalar a los estudiantes como los responsables de la misma. En esta disyuntiva hay ciertas disposiciones que deben ser internalizadas, es decir, algunos rituales que son considerados formas válidas y deseables de convivencia escolar. El respeto, la tolerancia, la amabilidad, la cordialidad, etc., son tan sólo algunos valores que circulan en torno a las lógicas de ser y estar en lo escolar (Flores, Sánchez, 2011). La convivencia, promoción de valores y cultura de paz, así como la resolución positiva de conflictos, enfatizan la cuestión ética y moral que no puede dejarse de lado y puede ser guía, orientación y marcos que promuevan una sana convivencia. No obstante, estas lógicas olvidan su naturalización normalizadora y normativizadora, que muchas veces es ajena a las formas de interacción que se puedan dar fuera de los límites escolares.

Es aquí donde el concepto de juego de lenguaje (Wittgestein, 1988) permitiría explicar un poco mejor esta lógica. Dentro de los entornos sociales, los jóvenes han constituido determinados habitus que, si bien se pudieron iniciar dentro de la familia en cooperación con la escuela en esos primeros años, en la actualidad el acceso a otras fuentes de información y, sobre todo, formas de socialización, ha permitido la instauración de otras visiones, a través de modos de expresión que van desde la música hasta las opciones de entretenimiento. El hecho es que la escuela escasamente ha modificado sus estructuras, en tanto que el contexto de los jóvenes ha sufrido cambios rápidos y significativos en los últimos años. En este sentido, se podría hablar de la construcción o existencia de diversos juegos de lenguaje que muchas veces se contraponen con las culturas instauradas desde el espacio escolar. Cabe señalar que, si bien es muy probable que los contextos -es decir, los diversos juegos de lenguaje desde los cuales se han constituido las subjetividades de los jóvenes estudiantes- pueden no ser congruentes con formas de ser y estar en el espacio social, lo cierto es que la gubernamentalización escolar ha mostrado en los últimos años una posible baja en su fuerza interpelatoria. Esto puede ser visible en los niveles de abandono escolar que parecen aumentar día a día sin que haya una propuesta que ayude a contener a los jóvenes en las aulas.

Este marco general que hasta ahora se ha intentado dibujar, refleja este complejo entramado y algunas preocupaciones, pero, sobre todo, cuestiones que posiblemente no han sido planteadas o más bien, no han sido tan visibles y, por ende, poco han ayudado a transformar los espacios que hoy por hoy están sesgados de diversos tintes de violencia. En este sentido, lo que se ha señalado se puede acercar a lo que implica un dilema, una tensión en la que se contra- 
ponen posturas o valores y cuyo reconocimiento en las estructuras escolares es necesario para comprender dicha complejidad (Dyson, Millward, 2000).

Por un lado, la escuela, para seguir reproduciendo su estatus de lugar en donde se forma la ciudadanía del mañana, ha echado mano de sus recursos simbólicos, pero también políticorepresivos, para intentar interpelar y disciplinar a los cuerpos que habitan día a día por varios años sus espacios áulicos. Por otra parte, la convivencia de los jóvenes ha cambiado y por ende sus posicionamientos; por ejemplo, en sus contextos particulares, la asistencia a otros posibles escenarios, la reflexión sobre otras lógicas de pensar y el acceso a literatura diversa que proviene centralmente de las propias comunidades y de los espacios sociales de la internet y demás lugares de socialización, hoy por hoy son hegemónicos por su capacidad de representación.

Una de las opciones que aparece en la literatura implica constituir sujetos políticos (Treviño, 2000), es decir, participativos, cual agentes que intervengan crítica y propositivamente; sin embargo, dicha operación implica para la escuela la pérdida de cierto poder y la flexibilización de las formas para relacionarse; la horizontalidad del acto en el intento de ejercer un reconocimiento de la juventud, no como sujetos carentes o en falta, sino como personas con voluntad que son capaces de ejercer su libertad y expresarla. De tal suerte que el intento de operar esta lógica, sin duda transformaría la estructuralidad impuesta y modificaría incluso hasta las propuestas curriculares.

Lo que este trabajo busca señalar es la ausencia de dicho dilema. Situación que se ha planteado desde diversos ámbitos cercanos al tema de la violencia social, pero que en el plano educativo se ha posicionado en la versión de la violencia entre pares, de una violencia física que sólo existe en la medida que puede verse materializada en determinados actos que sobre todo realizan los jóvenes, cuyas causas se encuentran en la ausencia de valores, principios educativos y normas que deberían haberse dado en el plano familiar, y en problemáticas de salud mental.

El problema es que este dilema parece no hacerse presente en los profesores, es decir, la situación violenta no es dilemática, no hay conflicto, los jóvenes están habitados por la violencia, la institución escolar es un espacio que ayudará a disminuir la violencia, a fortalecer las relaciones entre los jóvenes, a enseñarles a convivir, a fortalecer y favorecer los valores sociales. En otras palabras, es una institución aséptica, no violenta, instaurada desde la verdad para constituir verdades y, con ello, pensada como el remedio posible. En este caso, los profesores y profesionales de la educación no han puesto bajo sospecha la figura del aparato escolar. Este dilema de la violencia escolar no se ha declarado con todas sus letras desde dichos espacios $y$, al no hacerlo, escasamente ha contribuido a la solución del problema.

En este sentido, la pregunta ya no es por la posibilidad de si la escuela es capaz de resolver los problemas de violencia escolar, sino por la ausencia de una autocrítica en torno al papel que le ha tocado desempeñar, no sólo desde su aspecto normativo y normalizador, sino desde su acción pedagógica que, de entrada, refiere a una violencia simbólica — para muchos un mal 
necesario-, pero que al no ser objetivado para su revisión, sólo ha permitido la constitución de un campo de visibilidad donde son los estudiantes y sus nuevas culturas, sus representaciones y ausencia de valores, lo que provoca, causa y aumenta la violencia en la escuela. Esta mirada, que se impone como violencia simbólica al concebir al joven en un estado fallido, al expulsarlo de la sociedad negando su identidad pero que se formula como un acto de autoexclusión, no ha hecho más que negar otras causas y llevar a que el problema se replique sin fin.

En este sentido, tendría que hablarse de una violencia educativa, es decir, una violencia que se presenta en las tensiones entre una acción pedagógica determinada y unas subjetividades que poseen ya voluntades e intereses que, frente a las disposiciones escolares, deben ser ajustados o asimilados a tal grado que, de no hacerlo, la misma maquinaria emplaza a la producción de patologías, racionalidades violentas e individualidades antisociales.

Por tanto, se considera necesario indagar sobre las construcciones simbólicas de los profesores, para identificar los posicionamientos, orientaciones y omisiones que en su experiencia han constituido y, con ello, posibilitar otras formas de abordaje. En este sentido, a continuación se presentan los resultados de un estudio en torno a las significaciones que han construido profesores de educación media superior del estado de Puebla, México.

\section{Metodología}

El trabajo fue un estudio cualitativo de corte descriptivo cuya técnica fue el cuestionario cualitativo. Dicho instrumento fue sometido a un proceso de validación que constó de un jueceo realizado por tres expertos en el tema y un piloteo que se realizó con diez profesores de educación medida superior. El resultado de este proceso fue la reducción de diez a siete las preguntas del cuestionario, ajustes en la redacción y mayor especifididad de las preguntas (véase cuadro 1).

La aplicación del cuestionario fue en el marco de una serie de talleres sobre el tema de la violencia escolar; se aplicó a profesores de bachillerato con el propósito de recopilar información sobre los significados que los profesores tenían acerca de la violencia escolar y las posibilidades de la acción pedagógica para reducirla. Fue diseñado ex profeso e incluyó siete preguntas abiertas para recuperar las concepciones de los profesores, así como preguntas cerradas para los datos sociodemográficos. Cabe señalar que, aunque el cuestionario es un instrumento utilizado centralmente en estudios de corte cuantitativo, es un recurso metodológico que puede ser aplicado a una mayor cantidad de personas comparándolo con la entrevista, y que debido a la forma abierta de plantear las preguntas, permite acceder a ciertos campos simbólicos, como es el plano de los significados. 
Cuadro 1. Dimensiones

\begin{tabular}{|c|c|c|}
\hline Dimensiones & Definición & Preguntas \\
\hline $\begin{array}{l}\text { Significados de la violencia esco- } \\
\text { lar }\end{array}$ & $\begin{array}{l}\text { En esta dimensión se indagó so- } \\
\text { bre la información que los pro- } \\
\text { fesores tenía de la violencia, sus } \\
\text { definiciones, tipologías y clasifica- } \\
\text { ciones. }\end{array}$ & $\begin{array}{l}\text { Para usted, ¿qué es la violencia } \\
\text { escolar? } \\
\text { ¿Cuáles son los tipos de } \\
\text { violencia que conoce? }\end{array}$ \\
\hline $\begin{array}{l}\text { Manifestaciones de la violencia } \\
\text { escolar }\end{array}$ & $\begin{array}{l}\text { Esta dimensión indagó sobre las } \\
\text { creencias de los profesores sobre } \\
\text { las principales causas de la violen- } \\
\text { cia escolar. }\end{array}$ & $\begin{array}{l}\text { ¿Cuáles son las formas de } \\
\text { violencia escolar que más se } \\
\text { han manifestado en su institución } \\
\text { educativa? } \\
\text { ¿Cuáles considera son las } \\
\text { causas por las que se manifiesta } \\
\text { la violencia escolar en la actuali- } \\
\text { dad? }\end{array}$ \\
\hline $\begin{array}{l}\text { Formas y estrategias de interven- } \\
\text { ción desde la escuela }\end{array}$ & $\begin{array}{l}\text { Esta dimensión indagó sobre el } \\
\text { papel que juega la escuela para } \\
\text { hacer frente a la violencia. }\end{array}$ & $\begin{array}{l}\text { ¿Cuáles son las estrategias } \\
\text { que ha utilizado la institución } \\
\text { donde labora para minimizar la } \\
\text { presencia de violencia escolar? } \\
\text { ¿Cree que la escuela es un } \\
\text { lugar donde se puede ayudar } \\
\text { a disminuir la violencia que se } \\
\text { vive en el país? ¿Por qué? } \\
\text { ¿Qué opinión tiene acerca de } \\
\text { la siguiente afirmación? La } \\
\text { escuela es un lugar que ejerce } \\
\text { violencia cuando clasifica al } \\
\text { estudiante por medio de la } \\
\text { evaluación, apoyándose en } \\
\text { una serie de recursos que } \\
\text { terminan por estigmatizarlo. }\end{array}$ \\
\hline
\end{tabular}

Fuente: elaboración propia.

En el estudio participaron 57 profesores de educación media superior, seleccionados por conveniencia. $73.7 \%$ de los participantes fueron profesoras mientras que $23.3 \%$ profesores, que fueron clasificados en los siguientes rangos de antigüedad:

Tabla 1. Antigüedad docente

\begin{tabular}{|c|c|}
\hline Antigüedad & Cantidad de profesores \\
\hline $1-5$ años & 14 \\
\hline $6-10$ años & 16 \\
\hline $11-15$ años & 11 \\
\hline $16-20$ años & 6 \\
\hline
\end{tabular}




\begin{tabular}{|c|c|}
\hline $21-25$ años & 4 \\
\hline $26-30$ años & 4 \\
\hline $31-35$ años & 2 \\
\hline
\end{tabular}

Se realizó un proceso de codificación en donde $\mathrm{P}$ significa profesor y se colocó el número de cuestionario de acuerdo al orden en que fue devuelto.

Para el análisis de los datos, se construyeron categorías mediante un proceso de codificación abierta. Las preguntas del cuestionario giraron en torno a tres dimensiones analíticas: 1) significados de la violencia escolar; 2) manifestaciones de la violencia escolar y 3) formas y estrategias de intervención desde la escuela, las cuales se construyeron con base en los significados de los profesores. A continuación, se presentan los resultados de la aplicación de la técnica, así como su análisis.

\section{Análisis de los resultados}

Como ya se ha mencionado en este trabajo, las formas, tipos y expresiones de la violencia son diversos. Por ejemplo, para los profesores participantes, la violencia que ubican está íntimamente ligada a las formas en que su experiencia como docente les ha permitido identificarla e incluso actuar en consecuencia. Es así como, para ellos, la violencia puede expresarse en tres grandes dimensiones. Por una parte, se puede hablar de una violencia visible, que se encuentra en determinado estado material y que es posible ser captada por los sentidos; por otra parte, existe un tipo de violencia que es más sutil, pero no por ello menos problemática, que está inserta en las estructuras cognitivas, donde puede hacer el peor de los daños. Y finalmente, hay una tercera dimensión que se considera de mayor complejidad, en el sentido de que parece articular las dos dimensiones anteriores, para hacer referencia al bullying y ciberbullying, en donde no es tan clara la diferencia entre el daño físico y el psicológico que puede causar.

Tabla 2. Tipos de violencia

\begin{tabular}{|l|l|c|}
\hline \multicolumn{1}{|c|}{ Por su tipo } & $\begin{array}{c}\text { Por su manifestación en la } \\
\text { escuela }\end{array}$ & Número de veces que aparece \\
\hline Violencia física & Golpes & 49 \\
\hline Violencia verbal & Burlas, insultos & 30 \\
\hline Violencia sexual & Noviazgo & 39 \\
\hline Violencia psicológica & Apodos, discriminación & 3 \\
\hline Bullying & Acoso a algunos estudiantes & 8 \\
\hline Ciberbullyng & Acoso en redes sociales & 39 \\
\hline
\end{tabular}

Nota: Elaboración propia a partir de las respuestas dadas por los profesores de bachillerato. 
Por otra parte, los profesores señalan que las causas de esa violencia escolar son múltiples, sin embargo, podrían ser organizadas en dos dimensiones centrales: aquellas que son inherentes al estudiante, es decir, lo que tiene que ver con su subjetividad e identidad, y lo que parece provenir desde la exterioridad, sobre todo del contexto familiar, aunque el social y económico también es reconocido como espacio de producción de violencia. Cabe señalar que el lugar de lo familiar es donde más se hace evidente la supuesta génesis de la violencia; sin embargo, a esta representación le siguen las cuestiones individuales y psicológicas propias de los estudiantes que, como ya se había anotado en este trabajo, representa también un campo explicativo y productivo de condiciones de violencia escolar, en las que el estudiante y su ambiente familiar son los ámbitos donde se gesta esta problemática.

Tabla 3. Causas de la violencia escolar

\begin{tabular}{|l|c|}
\hline \multicolumn{1}{|c|}{ Causas } & Número de menciones \\
\hline Contexto familiar & 28 \\
\hline Contexto social y económico & 19 \\
\hline Problemas personales psicológicos & 26 \\
\hline Ausencia de valores & 10 \\
\hline Redes sociales e internet y medios de comunicación & 3 \\
\hline Falta de información y comunicación & 4 \\
\hline
\end{tabular}

Nota: Elaboración propia a partir de las respuestas de los profesores.

En este escenario, los profesores y las instituciones han planteado una serie de propuestas y estrategias para hacer frente a esta problemática. Dichas formas de intervención pueden ser clasificadas en tres grandes lógicas: una de ellas podría denominarse "pedagógica", en donde se busca que, por medio de talleres, charlas y conferencias, los estudiantes reflexionen sobre el problema, de tal suerte que se inicie un cambio a partir de las transformaciones internas que ellos realicen. Por otra parte, están las estrategias que podrían nombrarse "policiacas", en donde el uso de los reglamentos y el aumento de la vigilancia y el control representan las formas en que la violencia escolar puede reducirse. En una tercera lógica, se encuentra una serie de estrategias "Psi" en donde el trabajo es individual, se busca trabajar con los estudiantes desde una intervención psicológica y dinámicas particulares que reducen la mirada a los sujetos centrales en donde la violencia se ha hecho presente. 
Tabla 4. Estrategias para hacer frente a la violencia escolar

\begin{tabular}{|l|c|}
\hline \multicolumn{1}{|c|}{ Estrategia } & Número de menciones \\
\hline Pláticas y conferencias a los estudiantes & 28 \\
\hline Talleres con dinámicas vivenciales & 7 \\
\hline Uso de la normatividad: sanciones & 6 \\
\hline Platicar con los padres de familia & 17 \\
\hline Trabajo con agresor y agredido & 9 \\
\hline Intervención psicológica & 14 \\
\hline Vigilancia & 3 \\
\hline Campañas & 6 \\
\hline
\end{tabular}

Nota: Elaboración propia a partir de las respuestas de los profesores.

En general, es visible la dispersión en torno a causas, tipos y formas de abordaje en el tema de la violencia escolar, lo que puede servir de marco explicativo para analizar los significados que están presentes y han sido construidos a partir de las experiencias y vivencias que los profesores han tenido en el ámbito escolar.

\section{Significados en torno a la violencia escolar}

En general, los profesores participantes reconocen que la escuela es un lugar donde se puede trabajar la violencia escolar $y$, como ya se ha señalado, las intervenciones y acciones pueden ser diversas e inclusive ajenas a la escuela. No obstante, se muestra la posibilidad de incidir de alguna forma en este fenómeno educativo, el cual parece preocupar a más de uno de los participantes. Estas miradas sin duda responden a la idea o significado que sobre la violencia tienen los propios profesores.

Tabla 6. La escuela como un lugar para hacer frente a la violencia

\begin{tabular}{|c|c|}
\hline Respuesta & Número de veces que se menciona \\
\hline Afirmativa & 46 \\
\hline Negativa & 3 \\
\hline Parcialmente & 8 \\
\hline
\end{tabular}

Nota: Elaboración propia a partir de las respuestas de los profesores.

A continuación, se abordan estas referencias que permitieron la construcción de seis categorías analíticas divididas en tres dimensiones: 
- Dimensión intención racional/irracional: 1) violencia racionalizada; 2) violencia como enfermedad, y 3) violencia como acto unidireccional.

- Dimensión producción sujeto/subjetividad: 4) violencia como daño o perjuicio, y 5) violencia sentida.

- Dimensión génesis/causalidad: 6) violencia como reactividad familiar y social.

\section{Dimensión intención racional/irracional}

Los significados en torno al concepto de violencia escolar responden de entrada a una lógica multireferencial, es decir, en su interior conviven una serie de principios explicativos, lógicas de razonamientos y, sobre todo, conductas y personificaciones. En este sentido, los profesores dan cuenta del fenómeno, pero, también, muchas veces lo ubican cual entidad discreta que trastoca otros elementos como son: los asuntos familiares, la historia de vida de los estudiantes, los contextos sociales en los que conviven, e incluso, las posibles presencias de problemas personales a nivel psicológico.

Desde esta perspectiva, los significados en torno a la violencia escolar muestran la presencia de una intención y, con ello, de una racionalidad que precede al mismo acto. En otras palabras, un tipo de violencia racionalizada que implica una planificación anterior que dota al acto de cierta condición consciente que, con toda claridad, es realizada, planificada e incluso valorada por el sujeto que la ejerce. Algunos profesores señalaron:

\section{P-34. Es cualquier acto de tipo físico, verbal, psicológico que se realiza con la intención de dañar a algún} miembro de la comunidad escolar.

$\mathrm{P}-53$. Actos de agresión que van dirigidos de manera intencional hacia una o varias personas dentro del ámbito escolar.

P-12. Acciones con intención de dañar al alumno.

P-34. Intención de dañar a alguien de la comunidad educativa de forma psicológica, física o moral.

P-18. Es una acción que se ejerce en contra de alguien con la intención de dañar, siendo de diferentes tipos y modalidades.

P-2. Es la intención de agredir física, verbal o emocionalmente a algún miembro de la comunidad estudiantil, sea maestro, alumno, administrativo, de manera grupal o individual, dentro o fuera de una institución educativa.

P-11. Es la intención de dañar a los miembros de la comunidad escolar ya sea física o verbalmente.

Desde esta serie de representaciones, la violencia aparece ahí donde la intención se muestra y la finalidad es claramente un daño, por lo tanto, la violencia así entendida puede tener su génesis de actos predeterminados de odio, en la presencia de un tipo de maldad, la cual se hace presente en el sujeto. 
Por otra parte, frente a la idea de pensar la violencia escolar como un acto premeditado que responde a una racionalidad tal que permite el cálculo y la medición del daño, y que es plenamente consciente, y por ende razonada, se encuentra un significado de violencia que podría considerarse diferente al anterior. Un tipo de acto que, si bien es realizado por un sujeto, no pasa por una racionalidad, no al menos una que pueda considerarse consciente, atravesada por dicha medición y cálculo, por lo tanto, un acto en donde no se sabe a ciencia cierta si se realiza con la intención de causar un daño o si éste más bien es un resultado no previsto ni calculado. La violencia, entendida desde esta perspectiva, más bien pasa a ser considerada como una cuestión patológica, como una enfermedad que, habitando la subjetividad y el cuerpo del agresor, lo lleva a que de forma inconsciente o sin tener toda la claridad, realice un acto cuyo resultado será la agresión a otra subjetividad; se podría decir, un tipo de violencia entendida como enfermedad. En esta línea los profesores señalaron:

P-8. Alteraciones emocionales que se ejercen entre los miembros de una comunidad que pueden ser de diversa índole, físicos o emocionales.

P-57. Un impulso irracional. No controlado.

P-33. Es una forma de acción intencionada para dañar a otras personas, sea verbal, psicológica o física.

P-40. Es una convivencia poco sana entre los integrantes de la comunidad que integra la escuela, incluye alumnos, maestros, trabajadores escolares; donde por medio de acciones que trastoca la armonía, agreden a otros, obstaculizando el objetivo de la educación para la víctima dentro del ámbito escolar.

Si bien, desde esta forma de violencia el daño está presente y se comete de un sujeto a otro, el no control, la irracionalidad, la inconsciencia hacen que la violencia pueda ser entendida como un acto que se encuentra del lado de lo patológico, de lo enfermizo y por ende, la respuesta tenga que ser un trabajo individualizado y, en casos extremos, la expulsión de la escuela, pues definitivamente, sólo la intervención de los especialistas en salud mental podrá contribuir a una mejoría en el ahora "paciente".

\section{Dimensión producción sujeto/subjetividad}

Por otra parte, los significados de la violencia no están atravesados por la acción, sea o no consciente, sino más bien lo que produce; es así como para algunos profesores el foco de la violencia, su núcleo duro, está en lo que hace a otros. Desde este marco, es entendida no tanto desde la lógica de quien la realiza, la piensa, la planifica, sino desde quien la siente. La violencia se manifiesta ahí donde las heridas aparecen, donde las susceptibilidades se hacen presentes, en donde alguien se concibe herido y violentado, y con ello pueden desatarse una serie de emociones, miedos y desencuentros, que al final terminan dañando seriamente a la persona tanto física como emocional y psicológicamente, es decir, un tipo de violencia sentida. Por ejemplo: 
P-10. Es aquella que genera un malestar tanto al estudiante como al docente, regularmente se manifiesta de manera verbal o con lenguaje no verbal.

P-50. Es un daño a un miembro de la comunidad escolar, esto puede ser físico, emocional o verbal.

Por otra parte, de acuerdo con los participantes del estudio, la violencia escolar también es una acción que, sea quien sea quien la realice, atenta contra la integridad de una persona. Dentro de los límites escolares, dicha violencia puede estar presente entre los distintos agentes educativos; en este sentido, no es un acto exclusivo, por ejemplo, de los estudiantes, los profesores o demás personal que trabaja en la institución. Sin embargo, es una agresión que se sufre, que se padece, que lastima y lesiona, que representa un perjuicio y que puede estar ahí de forma material, pero también como un ejercicio de discriminación. La violencia escolar entendida como daño o perjuicio se recupera de los siguientes testimonios:

P-3. La agresión, física, verbal o psicológica que puede sufrir un alumno, docente, una persona administrativa, una persona de limpieza y cualquier compañero de trabajo que esté en el contexto escolar.

$\mathrm{P}-15$. El conjunto de acciones y conductas que se ejercen dentro de una institución educativa en perjuicio de un estudiante o estudiantes que lo dañan de manera física, emocional, social o psicológica. Ésta se puede ejercer de forma individual o en conjunto.

P-27. Es toda agresión o discriminación que se viva al interior de la comunidad escolar: estudiantes, profesores, padres de familia, directivos o personal no docente.

P-53. Las agresiones que se dan entre compañeros dentro de las aulas, bullying, discriminación, puede darse también entre compañeros de trabajo.

P-29. Es aquella agresión física, verbal y psicológica que se da entre miembros o círculos escolares.

P-38. Conductas agresivas e intimidantes dentro del contexto escolar.

Para otros profesores, la violencia escolar es un acto que, sin definir su racionalidad o inconsciencia, es realizado por alguien y dirigido hacia otra persona. En este sentido, implica una acción que va desde un sujeto a otro, si bien, desde esta perspectiva no es tan claro el motivo que mueve a los sujetos para realizar dicha acción, lo que es evidente es el tipo de relación que implica. Por ejemplo, en algunos casos se señala una asimetría en donde se puede buscar el control o la manipulación y, con ello, el emplazamiento de acciones cuyo interés puede estar cercano a la dominación del pensamiento y actuar del otro. En ambos casos, la violencia escolar es algo que se ejerce, pero que posee una direccionalidad y es posible ubicar su inicio y fin. Una violencia entendida como un acto unidireccional, tal como se recuperó de los profesores:

P-5. Es una agresión que se ejerce sobre una o varias personas afectando aspectos físicos o morales.

P-9. Es el tipo de agresión que se emite dentro de la institución y que afecta al estudiante física o psico-

lógicamente. 
P-57. Es una acción donde un individuo o grupo ejerce el uso de la fuerza para someter a otros en contra de su voluntad, la cual puede ser de forma física o psicológica.

P-46. Es una manifestación de comportamiento/conducta inapropiada en la cual una o más personas pueden agredir de forma física, verbal o psicológicamente a otros. A las personas que agreden se les llama victimarios o agresores y a los que son agredidos víctimas.

P-19. Es una conducta de agresión que una persona manifiesta a otra.

En otras palabras, desde las ideas de los profesores, la violencia es algo que se impregna contra una subjetividad e identidad y la hiere, lo que representa un uso de fuerza que termina menoscabando la dignidad de una persona.

\section{Dimensión génesis/causalidad}

En este marco, los participantes, dentro de este universo simbólico que representa para ellos la violencia, en sus significaciones han colocado algunos elementos causales, es decir, donde se da la génesis de la violencia. En el ámbito familiar es donde la violencia puede generarse, pues implica los primeros espacios de socialización. Para varios profesores, la existencia de violencia tiene que ver con una serie de normas morales que se han relajado al máximo. En otras palabras, la no existencia de la violencia se relaciona con una serie de elementos educativos que deben ser fraguados desde el ámbito familiar y comunitario cercano, asignando así una responsabilidad central a la acción del hogar en un problema que es más bien de actitud y educación. En este sentido, la violencia es entendida como reactividad familiar y social:

P-41. Es una actitud reflejada de una falta de valores y atención recibida desde casa.

P-28. La violencia escolar es una conducta con el fin de lastimar a alguien más, es la que se da en la escuela, las víctimas y agresores por lo tanto son estudiantes y ésta generalmente tiene que ver con la violencia que se ejerce en el seno familiar ya que los estudiantes reflejan lo que viven en casa. Cuando un estudiante ejerce violencia hacia otros estudiantes e incluso hacia docentes, muestran hacia los demás la violencia que quizá han sufrido en otro lado.

P-40. La representación de cualquier tipo de violencia que se vive en su núcleo familiar y social.

\section{Conclusiones}

Como se ha podido observar, los significados de los profesores dan cuenta de una gran variedad de formas y lógicas de entender la violencia escolar; entre ellas, son visibles algunas omisiones que van desde pensar que la violencia escolar es aquello que les es ajeno a su subjetividad, es decir, algo que está en los estudiantes y que se da entre los mismos: "Es aquella que se da en los jóvenes y que la manifiestan con acciones ya sean verbales o físicas ocasionando daño al joven [bullying]" (P-32), "Es cualquier acción realizada por un estudiante en contra de otro que 
lesiona su bienestar físico y emocional" (P-7), hasta elementos más elaborados como lo son el bullying y el ciberbullying; lo cierto es que los significados que la rodean no alcanzan a mostrar su auténtica complejidad. Las explicaciones parten de algunas suposiciones sobre los sujetos que realizan el acto de violencia, los sentires de la misma, las posibles causas, sus consecuencias; miradas todas que van de un punto a otro, que "pueden ser localizadas", y con ello se crea una cierta ficción sobre la posibilidad de aislarlas, ubicarlas, corregirlas, ejercer una intervención directa sobre ellas. Si bien estas formas de personificación y relación causal de la violencia son una realidad, también representan una mirada restringida acerca de la misma.

Hasta cierto punto, esta forma de entender la violencia escolar exclusivamente desde una determinada postura epistemológica permite ubicarla ahí donde puede ser vista, lo cual facilita actuar sobre ella; no obstante, resulta limitada en la medida en que sólo puede ver, por ejemplo, a quien la emite, la actúa y la ejerce, sin pensar que el acto de violencia escolar responde más a una serie de entidades discretas que se hallan en el contexto. Lo que puede llevar a separar a quien ejerce esa violencia para expulsar la "manzana podrida" que cohabita en los mismos espacios con los no violentos.

Dicha forma de entender la violencia escolar es limitada, en la medida que no permite pensarla dialécticamente como una malla que circula constantemente en espacios institucionalizados como la escuela, que no sólo implica un emisor y un receptor sino que da cuenta de una distribución reticular constante en donde no sólo aparece cuando da cuenta de su positividad, cuando se puede observar, sino que está también del lado de lo que se omite, de lo que está negado, incluso de aquello legitimado y puede ser considerado valioso para la institución escolar.

Habría que diferenciar entre la violencia en la escuela y la violencia de la escuela, sin embargo, lo que se pretende aclarar es que esta diferenciación no señala más que su carácter dialéctico, y con ello, complejo, donde la mirada no puede ser sólo desde la primera o segunda opción, sino que habría que entender que la violencia escolar es violencia en y desde siempre, es una relación entre lo que acontece y se materializa por los agentes, pero también lo que está instituido, lo a veces cotidiano, lo normado o incluso lo valioso.

En este sentido, se tendría que hablar de un tipo de violencia educativa, en donde el acto no puede ser pensado sólo en si los estudiantes y demás agentes ejercen violencia, sino en una relación constante y dinámica entre lo impuesto simbólicamente y la respuesta, consecuencia del mismo.

De esta forma, el dilema tendría que hacerse presente en las instituciones en donde se sospechara también que la violencia aparece, se manifiesta, se ejerce por cualquier agente, $y$, además, en el mismo acto educativo y en las tensiones que produce. En este sentido, la invitación es a no ver la presencia de este dilema como un componente negativo, sino más bien como una suerte de interrogación e indecibilidad constante que, en la medida que cuestiona la violencia en la institución, sus manifestaciones y sus desencuentros, también coloca la mira- 
da sobre la misma maquinaria escolar. Siendo así el ejercicio dialéctico inherente a la reflexión sobre el fenómeno. Por ejemplo, en la medida en que como profesores son capaces de pensar relacionalmente, es posible no sólo actuar sobre las situaciones que se consideran violentas, sino también conflictuar lo propio del sistema escolar.

Lo anterior puede llevar a un dilema entre lo que debe ser el acto escolar y lo que podría ser en las realidades vividas y experiencias situadas del espacio. En cierto sentido, el dilema se presenta entre aquello que debe ser impuesto como acto educativo, frente a lo que podría ser cambiado u omitido. Como se pudo observar, los significados de los profesores sólo dan cuenta de una parte de la fórmula puesto que la otra está obviada, dada, naturalizada y legitimada. La violencia escolar tendría que ser vista como lo impuesto por el mismo aparato escolar y las imposiciones, interacciones y disposiciones de los propios agentes que, entrando en contacto, pueden generar diversas formas de intercambios.

Todo parece suponer que en la medida que la violencia educativa no sea visible, sus efectos, producto de sus relaciones complejas con el todo, podrían seguir haciéndose presentes y las intervenciones seguirán las rutas marcadas sin que se llegue a resolver el problema, situación que tendría que pensarse ahí donde el dilema surge y donde se es capaz de cuestionar lo dado, lo institucionalizado, lo escolar.

En este marco, algunas discusiones posibles pueden estar ubicadas desde tres ámbitos o estratos. El primero, definitivamente es óntico-ontológico, donde se premie una reflexión sobre el ser de la escuela, sobre las existencias de los jóvenes al interior de la misma. Entendiendo la violencia, no como una entidad discreta desvinculada de una relación interseccional con otros elementos que facilitan su comunicación y presencia, sino la violencia como una relación articulada con toda la historia y experiencia de vida de las personas, con un carácter situado que la hace más visible y dañina en ciertos espacios y menos perceptible en otros.

Un segundo ámbito es que se requiere una discusión epistemetodológica que posibilite nuevas formas de mirar, aprehender y comprender el fenómeno; esto definitivamente requiere transformaciones no sólo desde las propias estrategias escolares donde se observa y se aborda, sino de las propuestas de investigación educativa que construyen ciertos discursos sobre la misma. Discursos que muchas veces no alcanzan a dar cuenta de la complejidad de su presencia, aparición e impacto, dando como resultado impresiciones y otras problemáticas que pueden terminar produciendo otras formas de violencia escolar.

Y una última discusión, sin duda es ético-política, sobre todo porque este fenómeno toca y trastoca lo humano, las subjetividades, las relaciones, las conductas, las emociones, haciendo que muchas veces la convivencia y la paz se vean como horizontes utópicos e inaccesibles. Pero, sobre todo, negando que los problemas de violencia son problemas de espacio, de normas, de ideales de plenitud que conviven con la naturaleza plural de lo social y que por ello implican 
una comprensión de la diversidad y diferencia de identidades, creencias, posiciones, e incluso, ideologías.

\section{Referencias}

Abella, V.; S. Barrio; D. Mango (2006). Asociación entre problemas de conducta y características de la personalidad en la adolescencia temprana. Revista de Psicología, 2(1), 131-140. https:// www.redalyc.org/pdf/3498/349832312011.pdf

Aznar, A.; D. González (2008). Capítulo dos. Análisis. En Aznar, A.; D. González. Son o se hacen: El campo de la discapacidad intelectual estudiado a través de recorridos múltiples (75-119). Argentina: Noveduc.

Bourdieu, P.; J. Passeron (1995). La reproducción. Elementos para una teoría del sistema de enseñanza. Barcelona: Fontamara.

Chuquilin, J.; M. Zagaceta (2017). La violencia en las escuelas desde la perspectiva de sus actores. El caso de una escuela secundaria de la Ciudad de México. Revista Educación, 41(2), 131-149. https://www.redalyc.org/articulo.oa?id=44051357009

Colom, J.; M. Fernández (2009). Adolescencia y desarrollo emocional en la edad actual. Revista de Psicología, 1(1), 235-242. https://www.redalyc.org/pdf/3498/349832320025.pdf

Del Tronco, J.; A. Madrigal (2013). Violencia escolar en México: una exploración de sus dimensiones y consecuencias. Trabajo Social UNAM, (4), 9-27. http://www.revistas.unam.mx/index. php/ents/article/viewFile/54048/48108

Dubet, F. (1998). Les figures de la violence a l'école. Revue Française de Pédagogie, (123), 35-45, http://www.inrp.fr/edition-electronique/archives/revue-francaisede-pedagogie

Fierro, C.; P. Carbajal (2019). Convivencia escolar. Una revisión del concepto. Psicoperspectivas. Individuo y Sociedad, 18(1). https://www.psicoperspectivas.cl/index.php/psicoperspectivas/ article/view/1486/982

Dyson, A.; A. Millward (2000). Schools and Special Needs. Issues of Innovation and Inclusion. Londres: Sage Publications.

Fajardo, F.; M. Fajardo (2010). Problemas educativos en los menores con trastornos de conducta. Revista de Psicología, 3(1), 447-455. https://www.redalyc.org/pdf/3498/349832326048.pdf

Flores, J.; E. Sánchez (2011). Educación en valores para el fortalecimiento de la convivencia escolar en el contexto educativo. Educare, 15(2), 151-167. https://revistas.investigacion-upelipb.com/index.php/educare/article/view/218/209

Foucault, M. (2002). Vigilar y castigar. Nacimiento de la prisión. Argentina: Siglo XXI.

Observatorio de la Juventud en Iberoamérica (2019). Encuesta de Jóvenes en México. México: Editorial SM. https://oji.fundacion-sm.org/encuesta-mexicana-de-la-juventud/ 
Galtung, J. (1990) La violencia, estructural, cultural y directa. Journal of Peace Reserch, 27(3), 391305.

INEGI (2015). Encuesta intercensal. México: INEGI. https://www.inegi.org.mx/programas/intercensal/2015/

García, P.; E. Armas (2008). Comorbilidad, personalidad, estilos educativos y problemas de conducta en adolescentes. Revista Anuario de Psicología Jurídica, 18(1), 21-30. https://journals. copmadrid.org/apj/art/ad3019b856147c17e82a5bead782d2a8

Gómez, A.; Ú. Zurita; S. López (2013). La violencia escolar en México. México: SINED-UCOL.

Gracia, E.; M. Fuentes; F. García (2010). Barrios de riesgo, estilos de socialización parental y problemas de conducta en adolescentes. Revista Intervención Psicosocial, 19(3), 265-278. https:// www.redalyc.org/pdf/1798/179817507007.pdf

Mansilla, M. (2000). Etapas del desarrollo humano. Revista de Investigación en Psicología, 3(2), 106-116. https://doi.org/10.15381/rinvp.v3i2.4999

Mayorga, C.; M. Godoy; S. Riquelme; L. Ketterer; J. Gálvez (2016). Relación entre problemas de conducta en adolescentes y conflicto interparental en familias intactas y monoparentales. Revista Colombiana de Psicología, 25(1), 107-122. https://doi.org/10.15446/rcp.v25n1.48705

Míguez, D. (2007). Reflexiones sobre la violencia en el medio escolar. Espacios en blanco. Revista de Educación, (17), 9-35. http://www.redalyc.org/pdf/3845/384539799001.pdf

Míguez, D. (2009). Las formas de violencia en las comunidades escolares. En Noel, G. (coord.). Violencia en las escuelas desde una perspectiva cualitativa (21-35). Argentina: Ministerio de Educación de la Nación.

Míguez, D. (2012). Cavilaciones epistemológicas sobre el estudio de la violencia en las escuelas. Elías y el equilibrio entre el nominalismo y positivismo. En Furlán, A. (coord.). Reflexiones sobre la violencia en las escuelas (72-101). México: Siglo XXI.

Justicia-Arráez, A.; G. Alba; M. Fernández; F. Justicia (2012). Reducción de problemas de conducta en educación infantil. International Journal of Developmental and Educational Psychology, 1(1), 257-266. https://www.redalyc.org/pdf/3498/349832342026.pdf

Organización de las Naciones Unidas (2016). Objetivos del Desarrollo Sostenible. https://www. undp.org/content/undp/es/home/sustainable-development-goals.html

Pérez-Sevilla, M. (2017). La violencia como enfermedad en México: las condiciones hegemónicas de la salud pública y sus figuras de poder-saber. Revista Pasajes, (5), 46-61. https:// revistapasajes.site/2017/11/ejemplar-5/

Pastor, Y. (2016). Perfiles de ajuste versus vulnerabilidad social en adolescentes españoles. Revista de Psicología, 25(1), 1-26. https://www.redalyc.org/pdf/264/26446630003.pdf

Romero, M.; A. Benavides; A. Quesada; G. Álvarez (2016). Problemas de conducta y funciones ejecutivas en niños y niñas de 5 años. Revista de Psicología, 1(1), 57-65. https://doi. org/10.17060/ijodaep.2016.n1.v1.214 
Salgado, R.; B. Fuentes; C. Escobar (2009). La desincronización interna como promotora de enfermedad y problemas de conducta. Revista Salud Mental, 32(1), 69-76. http://www.revistasaludmental.mx/index.php/salud mental/article/view/1269/1267

Saucedo, C.; C. Guzmán (2018). La investigación sobre la violencia escolar en México: Tendencias, tensiones y desafíos. Revista Cultura y Representaciones Sociales, 12(24), 213-245. http:// www.culturayrs.unam.mx/index.php/CRS/article/view/472

Skliar, C. (2005). Poner en tela de juicio la normalidad no la anormalidad. Falta de políticas en relación con las diferencias en educación. Revista Educación y Pedagogía, (41), 11-22. https:// revistas.udea.edu.co/index.php/revistaeyp/article/view/6024

Solé, J.; S. Moyano (2017). La colonización Psi del discurso educativo. Revista Foro de Educación, 15(23), 101-120. https://doi.org/10.14516/fde.551

Treviño, E. (2020). La violencia y lo político. Revista Clivajes, (13), 27-52. https://doi.org/10.25009/ clivajes-rcs.v0i13.2647

Trucco, D.; H. Ullmann (2015). Juventud: realidades y retos para un desarrollo con igualdad. Santiago: Naciones Unidas/CEPAL.

Vasen, J. (2011). Una nueva epidemia de nombres impropios. EI DSM-V invade la infancia en la clínica y las aulas. Buenos Aires: Noveduc.

Villa, M. (2011). Del concepto de juventud al de juventudes y al de juvenil. Revista Educación y Pedagogía, 23(60), 147-157. https://revistas.udea.edu.co/index.php/revistaeyp/article/ view/11421

Wittgestein, L. (1988). Investigaciones filosóficas. México: Editorial Crítica-Grijalbo. 